\title{
Localization of Tissues in High Resolution Digital Anatomic Pathology Images
}

\author{
Raja' S. Alomari ${ }^{a}$, Ron Allen ${ }^{b}$, Bikash Sabata ${ }^{b}$, and Vipin Chaudhary ${ }^{a}$ \\ ${ }^{a}$ University at Buffalo, SUNY, Buffalo, NY 14260 \\ ${ }^{b}$ Bioimagene Inc., Cupertino, CA 95014
}

\begin{abstract}
High resolution digital pathology images have a wide range of variability in color, shape, size, number, appearance, location, and texture. The segmentation problem is challenging in this environment. We introduce a hybrid method that combines parametric machine learning with heuristic methods for feature extraction as well as pre- and post-processing steps for localizing diverse tissues in slide images. The method uses features such as color, intensity, texture, and spatial distribution. We use principal component analysis for feature reduction and train a two layer back propagation neural network (with one hidden layer). We perform image labeling at pixel-level and achieve higher than 96\% automatic localization accuracy on 294 test images.
\end{abstract}

Keywords: Microscopy, anatomic pathology, parametric learning, digital pathology, HR color images.

\section{INTRODUCTION}

Digital anatomic pathology utilizes high resolution imaging technology for diagnosis of causes and effects of particular diseases. ${ }^{1}$ Our research utilizes the iScan ${ }^{\mathrm{TM} *}$ image acquisition, analysis, and archival system of BioImagene Inc. ${ }^{\dagger}$ Up to $160\{K \leq 160\}$ slides are loaded into the system racks. Each slide is automatically placed on the optical stage and color imaged at a resolution of $2286 \times 710$ pixels (Fig. 1). Then the crucial localization step, the focus of this research, produces two outputs: A set of high-resolution focus points $\left\{f_{i}: i \epsilon\left\{1 \ldots F_{k}\right\}\right\}$ where $F_{k}$ is the number of focus points in slide $k \epsilon\{1 \ldots K\}$ and bounding boxes for all tissue on the slide $\left\{b_{a}: a \epsilon\left\{1 \ldots B_{k}\right\}\right\}$ where $B_{k}$ is the number of

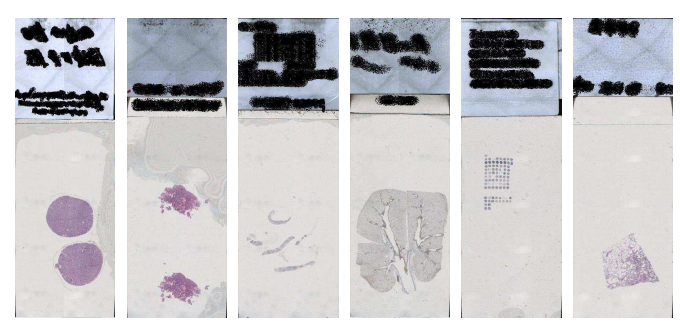

Figure 1. A sample set of slide images. The upper portion is reserved for label/bar code (blanked out for anonymity) and usually takes the top one-third of the image. non-overlapping tissue regions on slide $k \epsilon\{1 \ldots K\}$. A pre-scan is then performed using $F_{k}$ to determine tissue properties (height vs. sharpness). Then every frame in the boxes $B_{k}$ is scanned at high resolution. Image acquisition in iScan system is performed with two scanning resolutions: $0.46 \mu \mathrm{m} / \mathrm{pixel}$ and $0.23 \mu \mathrm{m} / \mathrm{pixel}$ with $20 \mathrm{X}$ and $40 \mathrm{X}$ power, respectively. A stitching operation is then performed on all these high resolution frames to produce the final high resolution image.

We focus on the localization step to achieve optimal outputs mentioned above: the set of focus points $\left(F_{k}^{*}\right)$ and the bounding boxes $\left(B_{k}^{*}\right)$. Because the set of focus points $\left(F_{k}^{*}\right)$ is responsible for determining tissue properties, the points should lie on tissues $T$ with minimal false positives as shown in Eq. 1 where $P(a \mid b)$ is the conditional probability that $a$ occurs given $b$. A false positive point wastes about 200 milliseconds. On the other hand, the second output, the bounding boxes $\left(B_{k}^{*}\right)$, should contain every useful tissue location with minimal false negatives (Eq. 2) so that the high resolution scan does not miss any tissue.

$$
\begin{aligned}
& F_{k}^{*}=\arg \min _{F_{k}} P\left(F_{k} \mid \bar{T}\right) \\
& B_{k}^{*}=\arg \min _{B_{k}} P\left(\bar{B}_{k} \mid T\right)
\end{aligned}
$$

Send correspondence to ralomari@buffalo.edu

${ }^{*}$ iScan ${ }^{\mathrm{TM}}$ is a product of BioImagene ${ }^{\circledR}$ Inc.

${ }^{\dagger}$ BioImagene ${ }^{\circledR}$ Inc. www.bioimagene.com, 1601 S. De Anza Blvd., Suite 212, Cupertino, CA, 95014. 
The localization saves both time and memory space during the scanning process. A slide takes about 1 hour for scanning without the localization step, while on average it requires about 15 minutes after applying successful localization. On the other hand, scanning the whole scannable region $\left(44.449 \mathrm{~mm} \times 25.4 \mathrm{~mm}=1290.3 \mathrm{~mm} \mathrm{~m}^{2}\right)$ at a sampling rate of $0.46 \mu \mathrm{m} /$ pixel $(20 \mathrm{X})$ produces more than 6150 high resolution images of dimensions $1024 \mathrm{x}$ 768 pixels. Considering overlaps between these high resolution images, which are used for the stitching stage, the final image dimension for one slide would be about 110,000 x 44,000 pixels which needs $110,000 \times 44,000$ x 3 bytes/pixel $=14.52$ GBytes. Successful localization reduces the required space to an average of $3 G B y t e s$.

\section{RELATED WORK}

Traditionally, image segmentation is defined as identifying regions $R_{k} \subset \Omega$ where $\Omega$ is the image to be segmented into regions $R_{k}$ and

$$
\bigcup_{k}^{N} R_{k}=\Omega
$$

where $N$ is the number of regions and $R_{j} \cap R_{k}=\emptyset, \forall j \neq k$. However, soft image segmentation softens the condition of hard subset to only one region by assigning probabilities for pixels to lie in regions.

Segmentation plays a significant role in medical imaging applications. Various methods have been applied in segmentation and delineation of anatomical structures and regions of interest in various medical imaging modalities. ${ }^{2-4}$ Early segmentation efforts used heuristic methods such as thresholding, edge detection and region growing. In these methods, the user needs to fine tune many parameters such as threshold values used as input to the segmentation algorithm.

The trend to pattern recognition methods such as Markov Random Fields, ${ }^{5}$ Neural Networks, graph based models, ${ }^{6}$ and deformable model ${ }^{7}$ started after many researchers found that heuristic methods are not feasible because of the high data dependency as well as the amount of effort for the user to fine tune all the required parameters. Markov Random Fields and Hidden Markov Models played a significant role in segmentation of soft organs such as the liver ${ }^{8}$ and most authors used deformable models for delineation of the boundaries. ${ }^{9,10}$

A third trend in medical imaging segmentation is based on incorporating knowledge based in forms of atlases, priors, or models. Atlas based segmentation ${ }^{11}$ is a popular method and it depends on the availability of an atlas for normal organs and a successful registration to the image under segmentation. Shape models also incorporate shape information ${ }^{12}$ for the organ of interest and sometimes the surrounding structures.

Neural networks (NNs) has been successfully used to segment various medical images. When ground truth is available (supervised learning), feed forward back propagation NNs are usually used. ${ }^{2}$ Several NN models for image processing problems have been proposed. ${ }^{13-16}$ Unsupervised NN was also used for segmentation problem to reduce the limitation of supervised learning such as Kohonens learning vector quantization (LVQ) network. ${ }^{17}$ Hall et al., ${ }^{13}$ perfomed a comparison between a Neural Network and fuzzy clustering techniques in segmenting MR Images of the Brain. They trained both methods on normal and abnormal cases and found that each method has advantages in some aspects. Unay et al., ${ }^{18}$ proposed an artificial neural network-based segmentation method. They apply their method on fruit images. They extracted statistical features at the pixel level, tested many classifiers, and achieved around 90\% recognition accuracy. Estevez et al., ${ }^{19}$ proposed a segmentation method for color images using fuzzy min-max neural network. ${ }^{20}$ Segmentation is similar to region growing but with only the seed point as input. They grow hyperboxes upto a limited size and can combine region if they obey specific homogeinity criteria.

In this paper we localize the tissue from the whole slide image so that further image analysis is perfomed only on the tissue. Many recent research efforts has been targetting segmentation, diagnosis, and prognosis problems in histopathology images. However, as far as we know, all of them target small sizes of the full HR image which is a zoomed piece of the whole slide image as shown in Fig. 2. We aim at indexing all the slide image and perfom further analysis on it by analyzing all subimages produced from the whole slide image rather than working on only pieces of the original image case.

Main research efforts in HR histopathology images have been focused on segmentation of anatomical structures and diagnosis of cancer cells. Latson et al, ${ }^{21}$ used basic fuzzy c-means clustering for nuclie segmentation from HR images while Petushi et al. ${ }^{22}$ used adaptive thresholding. However, these method do not extend for 


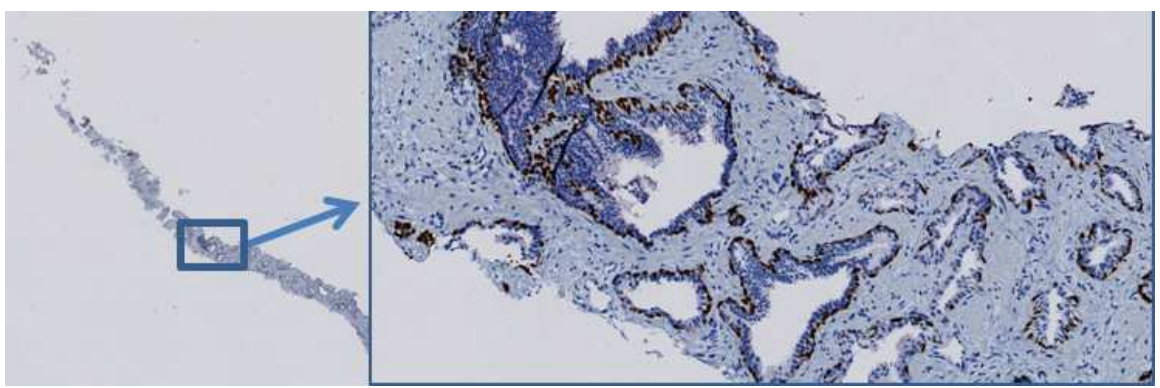

Figure 2. The whole slide image of prostate tissue (left) and a zoomed region (right).

high variabilities in data. ${ }^{23}$ Bamford et al., ${ }^{24}$ used active contours for segmentation of the nuclie which succeeds when there is no overlapping nuclie.

More recent work by Naik et al., ${ }^{23}$ proposed a two level approach for detection and segmentation of nuclie and glands from breast and prostate histopathology high resolution (HR) images. They used a Bayesian classifier for low-level likelihood generation, a high-level template for nuclie and level sets for gland detection, and domain knowledge integration for verification of detected structures. They perfomed grading of prostate and breast cancer as well as detection of malignant breast specimens. Other segmentation methods including segmentation of liver from $\mathrm{CT}^{9}$ and labeling of lumbar area discs in clinical $\mathrm{MR}^{25}$ has also been recently proposed.

\section{DATASET PREPARATION}

Because the main challenge in this problem is the high variability of images that need one solution for the localization problem, we collect a representative dataset for tissues in different shapes, sizes, counts, conditions of biopsy, appearances and colors. To clarify this we show a sample of this variability in Fig. 3. We use 294 different images to train, validate and test our proposed method. These images are collected from different pathology labs in two datasets containing 150 and 144 images.

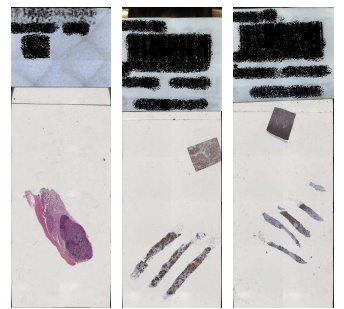

(a) Stained tissues

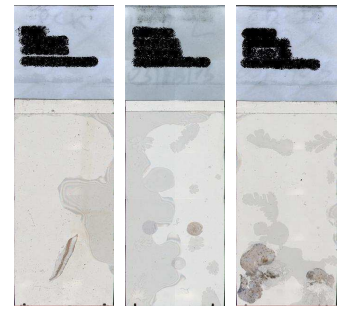

(d) Detritus in middle

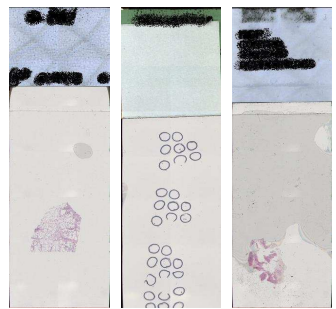

(b) Microscopic holes

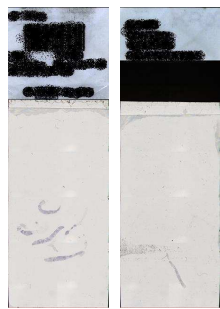

(e) Unstained
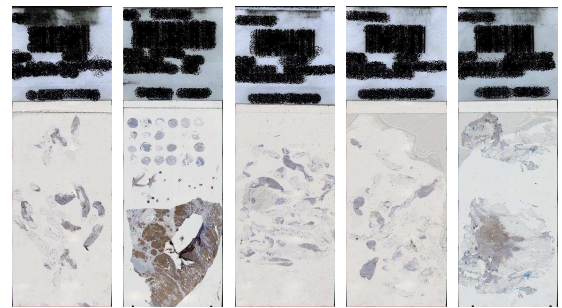

(c) Distriubuted pieces
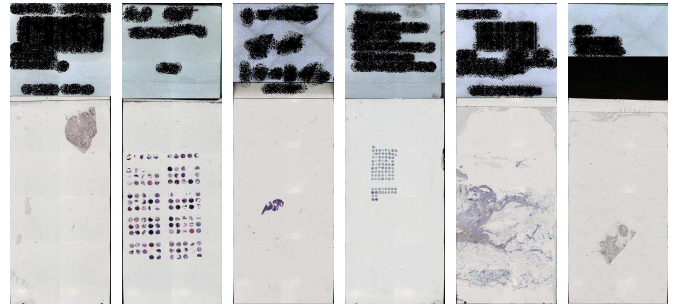

(f) Various shapes

Figure 3. Sample set showing the variability in shapes, sizes, counts, conditions of biopsy, appearances and colors of our dataset. The first set shown in Fig. 3(a) shows a well stained tissues that are easily distinguished from background using color. Fig. 3(b) shows some tissues with microscopic holes. Fig. 3(c) shows a set of tissues that are distributed all over the slide with multiple pieces. Fig. 3(d) shows how detritus move inside the slide image and interfere with the tissues. Fig. 3(e) shows thin strips of tissue that are unstained and appear very similar to the detritus. Fig. 3(f) shows variable shapes of tissues, the first and third are microarrays of tissues.

Upon examining the datasets, we found that tissues vary in some major characteristics including color, texture, intensity (appearance), count, and location. Most tissues have some sort of color but not all of them. 
As it is clear from Fig. 3, some tissues are just thin light gray strips in the middle of the slide image. Most tissues have texture that is distinguishable from the background, however, significant amount of tissues have no texture at all. Some tissues reside in one location (one piece) but some other (like the microarray tissues) have so many tissue pieces. Furthermore, tissues usually reside inside the slide but sometimes they might touch the border of the slide as well. The dataset is carefully created to include all these variabilities so that our method is correctly validated.

\section{METHOD}

Our method utilizes a back propagation feed forward neural network for labeling low-level pixel values as well as heuristic methods for feature extraction and fine tuning the results. Fig. 4 shows the general workflow in our method. The offline training phase trains a two layer neural network (with one hidden layer) on the features extracted from the training data. In order for the neural network to train efficiently, we perform pre- and post-processing steps: feature reduction using Principle Component Analysis (PCA), normalization of the features, and removal of redundant information. The online testing stage takes the resulting parameters (normalization factors, input layer weights, hidden layer weights, and biases) and performs testing at the pixellevel in new images. This testing stage contains pre- and post-processing steps including normalization of the feature vectors, median filters, and image filling. ${ }^{26}$

\subsection{Offline Training and Feature Extraction}

We obtain the training data by picking a set of points in each class (tissue, background, detritus). Each point is enclosed by a rectangular region of $20 \times 20$ giving a $21 \times 21$ window that is manually checked to totally lie inside one class. From each window (WSize $=21 \times 21$ ) the set of features are calculated producing one feature vector. Collectively, a set of training data is produced. Our training

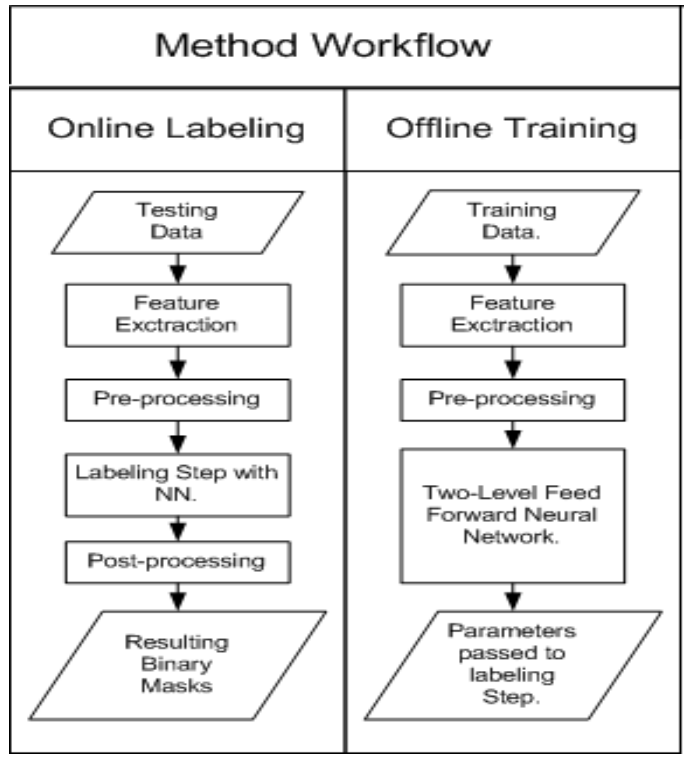

Figure 4. General Work Flow of our Method: Off line stage includes ground truthing, feature extraction and training of the Neural Network. On line stage takes parameters from the off line stage and produces a set of minimal false positive points and a minimal false negative bounding box. data is produced from 64 high resolution color images that were selected to represent all variability in data from different laboratories. This dataset contains: 1614 data point for the tissue class, 1106 data points for the detritus ${ }^{\ddagger}$ class, and 11983 data points for the background class.

Feature selection ${ }^{27,28}$ is a core step in any machine learning approach. Usually, there are two ways for selecting features; include a large number of features ranging from couple hundreds to a thousand, ${ }^{23}$ or carefully select a small set of features ranging from less than five to a hundred. ${ }^{29}$ We approach the problem by empirically examining a specific set of features representing the variabilities in the datasets. We start by empirically examining 25 features for color, intensity, and texture. Features that do not add significance to the learning process are rejected. In fact, we use PCA to reduce the feature vector besides the manual judgment of every feature.

Below we describe the effective features in each category:

\subsubsection{Color Features}

Color is an important feature because major portions of the tissue are often stained. However, some detritus contains color and color artifacts may be produced by reflection through filters present on the slide. These color artifacts are usually of pure colors. Stained tissues in our datasets appear with different colors in the images; mainly pink, green, brown, and blue with varying intensity levels. These colors are not pure colors, rather, they

\footnotetext{
${ }^{\ddagger}$ Detritus is particulate organic material that lies on slides because of lab conditions that is not related to the biopsy. Usually, it appears as gray material along sides and sometimes extends to the interior.
} 
appear more of mixed colors as shown in Fig. 3. This means that color features may not be specific to some color frequencies, instead, it should emphasize the meaning of color and the variability that is caused by color and distinguish it from the gray levels that represent the background and the detritus.

The main color feature is the variance in the three RGB channels, as both background and detritus have almost equal values for the three channels. We select these features to represent color information:

1. Variance $\left(\sigma_{\text {Peaks }}^{2}\right)$ between $P k \epsilon\{$ PeakR, PeakG, PeakB $\}$ where PeakR, PeakG, and PeakB are the peak values of color component in the Red, Green, Blue channels, respectively:

$$
\sigma_{\text {Peaks }}^{2}=\sum_{i=1}^{3}\left(P k_{i}-\mu_{P k}\right)^{2}
$$

where $\mu_{P k}$ is the mean value of the three peaks. This value is small in detritus and background due to the small variance in RGB values compared to stained tissues.

2. Variances $\left(\sigma_{R}^{2}, \sigma_{G}^{2}, \sigma_{B}^{2}\right)$ in RGB channels: $\sigma_{R}^{2}=\sum_{i}^{W \text { Size }}\left(R_{i}-\mu_{R}\right)^{2}, \sigma_{G}^{2}=\sum_{i}^{W \text { Size }}\left(G_{i}-\mu_{G}\right)^{2}$, and $\sigma_{B}^{2}=\sum_{i}^{W \text { Size }}\left(B_{i}-\mu_{B}\right)^{2}$ where $\mu_{R}, \mu_{G}$, and $\mu_{B}$ are the averages of R-, G-, and B-channel in the current window, respectively. The window size (WSize $=21 \times 21$ ) is constant. These three values are high in tissue and low in both the detritus and the background.

3. Mean hue value $\mu_{\text {hue }}$ in the HSI color model : $\mu_{\text {hue }}=\frac{1}{\text { WSize }} \sum_{i}^{\text {WSize }} H_{i}$ where $H_{i}$ is the hue value at location $i$ in the window. Because this value represents the color in HSI color model, it takes higher values in colored regions than gray regions (detritus and background). In fact, using the Hue does not replicate the color information from the RGB model according to our experimental analysis. We found that the addition of $a^{*}$ and $b^{*}$ from the $L^{*} a^{*} b^{*}$ color model does not add significant value to the results when we consider the high computational requirements.

\subsubsection{Appearance (Intensity) Features}

Intensity-based features are significant in two situations; capturing unstained tissues and capturing the white and gray microscopic holes inside the stained tissues. As we can see in Fig. 3, some tissues are unstained, thus, they don't appear with any color, rather, they are white to gray pieces inside the slide image. These tissues may not be captured by color features which necessitates adding intensity features. In fact, these tissues do not have any meaningful texture that might be captured by texture features.

On the other hand, in most stained tissues, some white and gray microscopic holes appear inside the tissue. These holes may not be captured because we label at the pixel level and closing or filling operation may not be useful because sometimes the tissues have holes that are not part of them such as circular tissues (Fig. 3).

In order to capture intensity information, we integrate both the mean gray intensity:

$$
\mu_{\text {gray }}=\frac{1}{W \text { Size }} \sum_{i}^{\text {WSize }} \text { gray }_{i}
$$

and the variance gray intensity $\sigma_{\text {gray }}^{2}=\sum_{i}^{W \text { Size }}\left(\text { gray }-\mu_{\text {gray }}\right)^{2}$ as part of the feature vector using the standard NTSC formula:

$$
\text { gray }=0.2989 * R+0.5870 * G+0.1140 * B
$$

where R, G, and B are the Red, Green, and Blue channels of the RGB color model, respectively. In fact, there are different formulae to gain intensity from color images such as the I in the HSI color model and the L in the $L^{*} a^{*} b^{*}$. However, the NTSC formula is a standard that gives meaningful intensity information. 


\subsubsection{Texture Features}

Texture also help in identifying tissue. Integration of some covariance matrix $C O$ statistics add significant enhancement to the localization process. $C O$ is the matrix of covariances between elements of a vector:

$$
C O=\sum_{i, j}^{\operatorname{Size}(C O)} E\left[\left(X_{i}-\mu_{i}\right)\left(X_{j}-\mu_{j}\right)\right]
$$

where $\mu_{i}=E\left(X_{i}\right)$ is the expectaion of the $i^{t h}$ entry in the vector $X .{ }^{30}$ We use Correlation and Energy which are defined as:

$$
\begin{gathered}
\text { Correlation }=\sum_{i, j}^{\text {Size }(C O)} \frac{(i-\mu i)(j-\mu j)(C O(i, j))}{\sigma_{i} \sigma_{j}} \\
\text { Energy }=\sum_{i, j}^{\text {Size }(C O)} C O(i, j)^{2}
\end{gathered}
$$

\subsubsection{Spatial Features}

Spatial features are extracted from the spatial distribution of tissues on the slides. These features mainly distinguish between the detritus and the colorless gray tissue that resides inside the slide image. In most cases, the detritus lies along the edges of the slide image and may extend to the inside of the image but in almost all cases it keeps its base touching the edges of the slide. However, tissue might sometimes touch the edges, thus, not everything touches the edges is detritus.

We use a heuristic method based preliminary segmentation to obtain this feature. A set of normalized features $\left(\sigma_{\text {Peaks }}^{2}, \sigma_{R}^{2}, \sigma_{G}^{2}, \sigma_{B}^{2}, \mu_{\text {gray }}\right.$, and $\left.\sigma_{\text {gray }}^{2}\right)$ are collectively used at the whole image level. This highlights the tissue region as every feature adds higher value for tissue pixels and lower values for other pixels (background and detritus). Then we apply the Otsu ${ }^{31}$ threshold algorithm to achieve preliminary segmentation. Next we apply connected component analysis to analyze this initial segmentation. The pixels of the components that are connected to the border (within 50 pixels) are assigned lower probability value (0.001) while the rest are assigned a higher value (0.5). We use this border map for post-processing the results obtained from the neural network. In fact, this feature is the key for localizing tissues containing gray thin strips; intensity-based features do not distinguish between this type of tissue and the detritus extending from the border.

\subsection{Neural Network}

We train a back propagation feed forward neural network ${ }^{32,33}(N N)$ that consists of two layers with 20 neurons for the hidden layer and one output neuron. The input layer has the same number of neurons as the input features vector size of 15 .

We perform preprocessing steps on the training data $\left(X j=<x_{i}>, 0 \leq i \leq 15,0 \leq j \leq\right.$ TrainDataSize $)$ to utilize the work of the NN; values scaling and removal of duplicate training data. The feature values in the training data are scaled to $x_{i} \epsilon[-1,1]$ and all duplicate feature vectors are then removed. The scaling factors are passed as parameters to the testing (labeling) stage to normalize the testing vectors as well.

Ground truth target values $T$ are provided at each window (21 x 21 pixels) in our training dataset which makes each window as a feature vector $\left(X=<x_{i}>\right.$ and $\left.g(X)=T\right)$. The Logistic transition functions $f(n)=\frac{1}{\left(1+\exp ^{-n}\right)}$ are used for both layers. The output $(O)$ is then mapped into one of the three classes (tissue, detritus, or background) using simple thresholding. The mean squared error is used as the performance function and the Levenberg-Marquardt algorithm is used for back propagation.

\subsection{Testing Stage}

Testing stage accepts new images and labels every pixel to one of the three classes. First, we divide the input image into equal size blocks (windows) and then extract the same feature vector from every window. Every feature vector $\left(V=\left\langle v_{i}\right\rangle\right)$ is preprocessed to scale the values $v_{i} \epsilon[-1,1]$ and then it is input to the testing stage of the NN. The threshold value for every class is then used to determine the class of the window that produced this feature vector. All pixels inside this window take the same class label. 
Table 1. Results on the training set and two test sets. Any image that shows any portion as false negative/positive is counted as false negative/positive. If this false negative/positive affects the localization mask, then it is counted as localization error. Our method achieves $96.9 \%, 94 \%$ and $96.5 \%$ localization accuracy on the three datasets.

\begin{tabular}{|c|c||c|c|c|c|}
\hline Dataset & Size & False Negatives & False Positives & Localization error & Localization Accuracy \\
\hline Training & 64 & 8 & 3 & 2 & $96.9 \%$ \\
\hline Testing & 150 & 13 & 9 & 9 & $94 \%$ \\
\hline Testing & 144 & 10 & 6 & 5 & $96.5 \%$ \\
\hline
\end{tabular}

One main implementation issue is the choice of the window size. During the training, we use $(20 \times 20)$ pixels for each window. However, in the testing stage, we may use any suitable size. If we choose coarse window sizes, the resulting image will appear blocky as all pixels inside the window will have the same class label. On the other hand, when we choose a fine window size, smoother results are obtained but more computation time is required. We found that $(5 \times 5)$ windows represents a good compromise between the computation time and acceptable results.

In our experiments, we use a $(5 \times 5)$ window size for running the neural network. We then apply a median filter (with $20 \times 20$ convolution window) on the resulting image, following an image filling ${ }^{26}$ operation. We then apply the border map to eliminate border connected components.

\section{EXPERIMENTAL RESULTS AND DISCUSSIONS}

Our dataset consists of 294 images collected in two datasets of 150 and 144 images. We use 64 images from the first set for extracting the data points for training as discussed in section 3 . Then we perform labeling on three sets: the 64 images, all 150 images, and the 144 images as shown in Tab. 1. Labeling the training data aims at showing comparative results with labeling of testing data.

In order to measure labeling accuracy that serves our target, we label all the images and produce binary masks that represent the tissue class. For these images, we overlay the binary masks on the corresponding images. Next, an expert examines every image and decides whether it has correct localization or not, and if it has false negative or false positive. The resulting image is correctly localizing the tissue if all tissue on the slide appears in the binary mask. Then the expert decides if there is false negative, i.e., some portions of the background (or detritus) is labeled as tissue, or if there is false positive, i.e., some portions of the tissue is labeled as background (or detritus). Note that the resulting image might have false positive and false negative regions at the same time. After this, the number of resulting images that have significant amount of either false positive or false negative are counted as localization error $\left(L_{\text {Error }}\right)$ and thus the whole image is considered as mislabeled. The accuracy; which appears in the last column of Tab. 1, is then

$$
\text { Accuracy }=\left(1-\frac{\text { Loc }_{\text {Error }}}{N}\right) * 100 \%
$$

where (Locerror) is the number of images incorrectly localized and $N$ is the total number of images in the dataset.

The training data (64 images) labeling produce $96.9 \%$ accuracy which means that only two images were considered mislabeled by the expert. According to Tab. 1, eight images have portions of false positive and three images have portions of false negative. However, only two images have significant amount of false labeling (either negative or positive or both) to be considered an error for localization.

In the next experiment, we label the entire first dataset of 150 images that includes the 64 training images. Only nine images contain significant amount of false labeling. In fact, thirteen images contain portions of false negatives and nine images contain false positives. It is worth mentioning that this does not mean that 22 images have problems because the same image might contain false negative and false positive pixles at the same time. The accuracy for this dataset is $94 \%$ which is below the training set by 3 percentage points. However, after examining the dataset we found two images with handwritten markers on top of the tissue region which caused the mislabeling.

The third experiment labels 144 images, none of which were used for training. Five images were marked to have localization error among the sixteen images that contain false negative (ten images) or false positive (six 
images). The overall accuracy is $96.5 \%$ which is around the same level as the dataset that includes the training data. In other words, the labeling on a randomly selected dataset is expected to be over $96 \%$.

Fig. 5 shows a set of test images (none from the training set) and the final results from our method. The resulting binary mask is overlaid on the original image for visual localization quality. As Fig. 5(a) shows, localization of the tissues is excellent with almost $100 \%$ accuracy and negligible false positives and negatives. All images that have localization similar to fthese images are considered correctly localized with neither false negative nor false postive. However, when you compare the resulting image with the original image you might see very small number of pixels that are incorrectly labeled; especially in the original resolution of the image, but this misclassification is negligible compared to the size of the image.

Fig. 5(b) shows some false negative/positive portions in microarray tissues due to the high variability in such tissues. Despite that these images are reported as false negative/positive because the size of the false negative/positive pixels is significant. They are reported as correctly localized because the portion of the false negative is not affecting the localization of the tissues. In other words, these two images are counted in the 3rd (and/or) 4th columns but not in the 5th column. Fig. 5(c) shows various tissue types that contain small false negatives. These are due to white microscopic holes in the tissue structure or thin tissues that are indistinguishable from border detritus. These results also do not affect the localization accuracy, but they count as false negatives in table 1. Fig. 5(d) shows an example of a localization error image. In fact, this image has significant portion of false negative because significant portion of tissue is labeled as background. The significance here is that the bounding box will not include all tissues because the tissue is spread all over the slide.
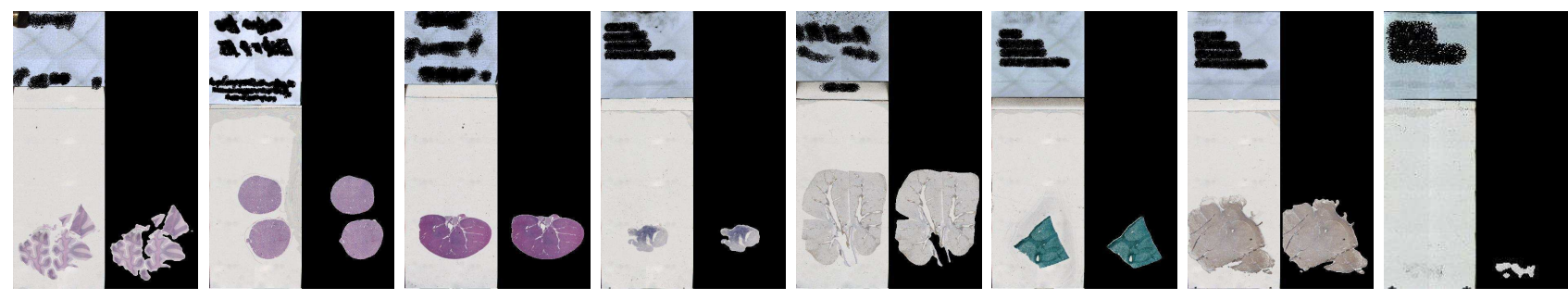

(a) Correct Localization of tissues.

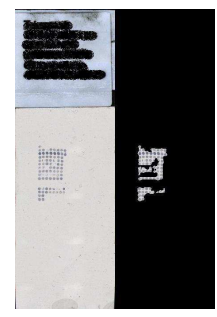

(b) Contains false negative.

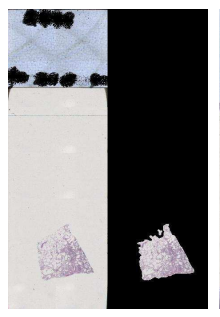

(c) Localization of various light stained cases.

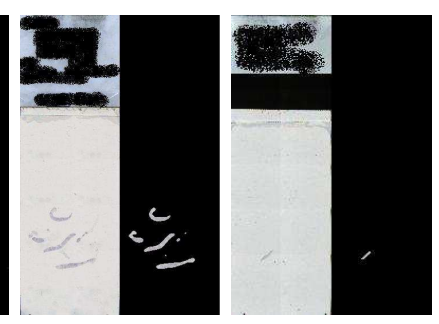

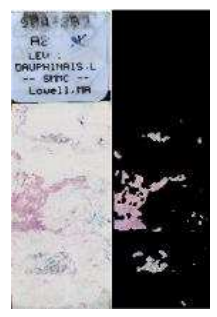

(d) Error

Figure 5. Classification results from our method. Original Image (Label portion is blanked for anonymity) and binary mask result overlaid on top of the original image for visual comparison. Fig. 5(b) and (c) contain some portions of false negatives/positives that are counted in false negative/positive columns of Table 1. Fig. 5(d) shows a sample case of an error in localization where the false negative portion is significant and affecting the localization decision of the tissue.

\section{CONCLUSION}

We proposed a systematic approach to solve the localization problem in pathology images. Our proposed a method utilizes a feed forward back propagation Neural Network with pre- and post-processing and many features including; color, texture, appearance, and location. We trained the NN on a large set of data points extracted from 64 images by manually selecting points inside these images. All these points with an enclosing (20 x 20) window lie in the same class. We have three classes, namely, tissue, detritus, and background. We produce 1614 data point for the tissue class, 1106 data points for the detritus class, and 11983 data points for the background class. The tissue is usually colorful with distinguished texture and lies inside the image. The detritus is usually noisy gray regions and the background is usually noisy white. However, exceptions for 
all these exist where we find unstained tissues, tissues touching the border, or tissues spread all over the slide image.

We tested the labeling procedure on 294 images and achieved over $96 \%$ labeling accuracy according to judgment from an expert pathologist.

\section{ACKNOWLEDGEMENT}

The authors would like to thank both the New York State Foundation for Science, Technology and Innovation (NYSTAR) and BioImagene Inc staff for their support.

\section{REFERENCES}

[1] Lefkowitch, J. H., [Anatomic Pathology], Saunders, New York City, USA (2006).

[2] J.C. Bezdek, L.O. Hall, L. C., "Review of mr image segmentation techniques using pattern recognition," Medical Physics 20(4), 1033-1048 (1993).

[3] Pham, D. L., Xu, C., and Prince, J. L., "A survey of current methods in medical image segmentation," Annual Review of Biomedical Engineering 2, 315-338 (2000).

[4] Withey, D. and Koles, Z., "Medical image segmentation: Methods and software," in [Proceedings of NFSI and ICFBI 2007], (Oct. 2007).

[5] Zhang, Y., Brady, M., and Smith, S., "Segmentation of brain mr images through a hidden markov random field model and the expectation maximization algorithm," IEEE Trans. Medical Imaging 20(1), 45-57 (2001).

[6] Corso, J. J., Yuille, A. L., Sicotte, N. L., and Toga, A., "Detection and Segmentation of Pathological Structures by the Extended Graph-Shifts Algorithm," in [Proceedings of Medical Image Computing and Computer Aided Intervention (MICCAI)], (2007).

[7] McInerney, T. and Terzopoulos, D., "Deformable models in medical image analysis: A survey," Medical Image Analysis 1(2), 91-108 (1996).

[8] Pham, M., Susomboon, R., Disney, T., Raicu, D., and Furst, J., "A comparison of texture models for automatic liver segmentation," in [Proceedings of the SPIE Medical Imaging 200\%: Image Processing.], Pluim, J. P. W. and Reinhardt, J. M., eds., 6512, 65124E (2007).

[9] Alomari, R. S., Kompalli, S., and Chaudhary, V., "Segmentation of the liver from abdominal ct using multifeatured markov random fields.," in [Proc. of the 3rd International Conference on Availability, Reliability and Security CISIS'08], 293-298 (2008).

[10] Philips, C., Susomboon, R., Mokhtar, R., Raicu, D., and Furst., J., "Segmentation of soft tissue using texture features and gradient snakes," in [Technical Report TR07-011], (2007).

[11] Cuadra, M. B., Pollo, C., Bardera, A., Cuisenaire, O., and guy Villemure, J., "Atlas-based segmentation of pathological mr brain images using a model of lesion growth cuadra," IEEE Transactions on Medical Imaging 23, 1301-1314 (Oct. 2004).

[12] Van Ginneken, B., Frangi, A., Staal, J., ter Haar Romeny, B., and Viergever, M., "Active shape model segmentation with optimal features," IEEE Transactions on Medical Imaging 21, 924-933 (Aug. 2002).

[13] Hall, L. O., Bensaid, A., Clarke, L., Velthuizen, R., Silbiger, M., and Bezdek, J., "A comparison of neural network and fuzzy clustering techniques in segmenting magnetic resonance images of the brain," IEEE Trans. Neural Networks 3, 672682 (Sept. 1992).

[14] Ghosh, A., Pal, N. R., and Pal, S. K., "Self-organization for object extraction using a multilayer neural network and fuzziness measures," IEEE Trans. Fuzzy Syst. 1, 5469 (Feb. 1993).

[15] Boskovitz, V. and Guterman, H., "An adaptive neuro-fuzzy system for automatic image segmentation and edge detection," IEEE Trans. Fuzzy Systems 10(2), 247-262 (2002).

[16] Reyes-aldasoro, C. C., Aldeco, A. L., and Mxico, A., "Image segmentation and compression using neural networks," in [Advances in Artificial Perception and Robotics CIMAT], 23-25 (2000).

[17] Pal, N., Bezdek, J., , and Tsao, E., "Generalized clustering networks and kohonens self-organizing scheme," IEEE Trans. Neural Networks 4, 549557 (July 1993).

[18] Unay, D. and Gosselin, B., "Artificial neural network-based segmentation and apple grading by machine vision," in [Proceedings of IEEE International Conference on Image Processing (ICIP)], 2, II- 630-3 (Sept. $2005)$. 
[19] Estevez, P., Flores, R., and Perez, C., "Color image segmentation using fuzzy min-max neural networks," in [Proceedings of IEEE International Joint Conference on Neural Networks (IJCNN)], 5, 3052- 3057 (Aug. 2005).

[20] Simpson, P. K., "Fuzzy min-max neural networks clustering," IEEE Transaction Fuzzy Systems 1(1), 32-45 (1993).

[21] Latson, L., Sebek, B., and Powell, K., "Automated cell nuclear segmentation in color images of hematoxylin and eosin-stained breast biopsy," Analytical and Quantitative Cytology and Histology 25(6), 321331 (2003).

[22] Petushi, S., Garcia, F., and Haber, M., "Large-scale computations on histology images reveal gradedifferentiating parameters for breast cancer," BMC Medical Imaging 6 (October 2006).

[23] Naik, S., Doyle, S., Agner, S., Madabhushi, A., Feldman, M. D., and Tomaszewski, J., "Automated gland and nuclei segmentation for grading of prostate and breast cancer histopathology," in [Proceedings of the IEEE International Symposium on Biomedical Imaging (ISBI)], 284-287 (2008).

[24] Bamford, P. and Lovell, B., "Unsupervised cell nucleus segmentation with active contours," Signal Processing 71, 203213 (1998).

[25] Corso, J. J., Alomari, R. S., and Chaudhary, V., "Lumbar disc localization and labeling with a probabilistic model on both pixel and object features.," in [Proc. of the 11th International Conference on Medical Image Computing and Computer Assisted Intervention (MICAAI 2008)], 202-210 (2008).

[26] Soille, P., [Morphological Image Analysis: Principles and Applications], Springer-Verlag, Berlin New York (1999).

[27] Squire, D., Muller, W., Muller, H., and Raki, J., "Content-based query of image databases, inspirations from text retrieval: inverted files, frequency-based weights and relevance feedback," in [Proceedings of 11th Scandinavian Conference on Image Analysis (SCIA'09)], 7-11 (1999).

[28] Philippe C. Cattin, Herbert Bay, L. V. G. and Szkely, G., "Retina mosaicing using local features," in [Proc. of Medical Image Computing and Computer Assisted Intervention (MICCAI'06)], 4191, 185-192 (Sep. 2006).

[29] Wang, J. Z., Li, J., and Wiederhold, G., "Simplicity: Semantics-sensitive integrated matching for picture libraries," IEEE Transactions on Pattern Analysis and Machine Intelligence 23(9), 947-963 (2001).

[30] Wasserman, L., [All of Statistics: A Concise Course in Statistical Inference], Springer Texts in Statistics, Springer (2004).

[31] Otsu, N., "A threshold selection method from gray-level histograms," IEEE Trans. on Systems, Man, and Cybernetics 9(1), 62-66 (1979).

[32] Rojas, R., [Neural networks: a systematic introduction], Springer-Verlag, Berlin New York (1996).

[33] Bishop, C., [Pattern Recognition and Macine Learning], Springer, New York (2006). 